\title{
Frecuencia de (BLEE) (AmpC) y CARBAPENEMASAS en muestras de urocultivo, en cepas de Escherichia Coli de origen comunitario
}

\author{
Frequency of (ESBL) (AmpC) and CARBAPENEMASES in urine culture samples, in Escherichia \\ Coli strains of community origin
}

\section{Freqüência de (ESBL) (AmpC) e CARBAPENEMASES em amostras de urocultura, em cepas de Escherichia Coli de origem comunitária}

\author{
Veronica Mendieta Astudillo \\ vmastudillo1993@hotmail.com \\ https://orcid.org/0000-0001-7506-6088
}

\author{
Juan Diego Gallegos Merchan \\ juandye@hotmail.com \\ https://orcid.org/0000-0001-6764-2911
}

\author{
Susana Janeth Peña Cordero \\ spenacordero@hotmail.com \\ https://orcid.org/0000-0002-6526-2437
}

\begin{abstract}
Programa de Maestría en Diagnóstico de Laboratorio Clínico y Molecular Neolab, Ecuador Universidad Católica de Cuenca, Cuenca, Ecuador
\end{abstract}

Recibido 29 de marzo 2021 | Arbitrado y aceptado 16 de abril 2021 | Publicado en 4 de mayo 2021

\begin{abstract}
RESUMEN
La Escherichia coli (E. coli) forma parte del grupo de las principales enterobacterias, que por su alta prevalencia y capacidad de generar resistencia a ciertos antimicrobianos es la causante de varios procesos infecciosos de origen comunitario sobre todo infecciones del tracto urinario. El mecanismo de defensa de estas bacterias es la activación enzimática a través de las Betalactamas de tipo BLEE, AmpC y CARBAPENEMASAS. Materiales y Métodos. Diseño documental transversal, con un enfoque cuantitativo, la población estuvo constituida por 671 informes de urocultivo procesadas en el período de enero - abril de 2020 en el Laboratorio Clínico Neolab de la ciudad de Cuenca en el Ecuador. Resultados. El 96,4\% de las muestras son de pacientes femenino y un 3.6\% al género masculino, el promedio de edad, el estudio tuvo mayor prevalencia en pacientes de 40 años de edad. El 7.62\% de las muestras resultaron BLEE positivas, $0.13 \%$ AmpC y el 0\% CARBAPENEMASAS. Los antibióticos con un mayor índice de efectividad para tratar la E.coli productora de BLEE en urocultivos fueron la Nitrofurantoína, la Gentamicina y Fosfomicina con índices de efectividad del $87.93 \%, 79,31 \%$ y 70,68\% respectivamente. Sin embargo los antimicrobianos como Ampicilina presentaron una resistencia del 100\% y Trimethoprim sulfa metoxazol del 72.41\%. Conclusión. La detección de frecuencia de enzimas BLEE, AmpC y Carbapenemasas, orienta a un tratamiento terapéutico adecuado. Los pacientes con bacterias productoras de BLEE, tienen un alto índice de mortalidad al ser tratados con antimicrobianos que las bacterias presentan una resistencia alta.
\end{abstract}

Palabras clave: Infecciones Urinarias; Farmacorresistencia Bacteriana; Pruebas de Sensibilidad Microbiana; Infecciones Comunitarias Adquiridas; Enzimas

\begin{abstract}
Escherichia coli (E. coli) is part of the group of the main Enterobacteriaceae, which due to its high prevalence and capacity to generate resistance to certain antimicrobials is the cause of several infectious processes of community origin, especially urinary tract infections. The defense mechanism of these bacteria is enzymatic activation through ESBL-type Betalactams, AmpC and CARBAPENEMASES. Materials and Methods. Cross-sectional documentary design, with a quantitative approach, the population consisted of 671 uroculture reports processed in the period January - April 2020 at the Neolab Clinical Laboratory in the city of Cuenca in Ecuador. Results. $96.4 \%$ of the samples are from female patients and 3.6\% from male gender, the average age, the study had a higher prevalence in 40 -year-old patients. $7.62 \%$ of the samples were positive ESBL, $0.13 \%$ AmpC and 0\% CARBAPENEMASES. The antibiotics with the highest effectiveness rate for treating ESBL-producing E.coli in urine cultures were Nitrofurantoin, Gentamicin, and Fosfomycin, with effectiveness rates of $87.93 \%, 79.31 \%$, and $70.68 \%$, respectively. However, antimicrobials such as Ampicillin showed a resistance of $100 \%$ and Trimethoprim sulfamethoxazole of $72.41 \%$. Conclusion. The frequency detection of ESBL, AmpC and Carbapenemases enzymes guides an adequate therapeutic treatment. Patients with ESBL-producing bacteria have a high mortality rate when treated with antimicrobials that the bacteria have high resistance.
\end{abstract}

Key words: Urinary Infections; Bacterial Drug Resistance; Microbial Sensitivity Testing; Community Acquired Infections; Enzymes
VM: Química Farmaceuta, Universidad Católica de Cuenca. Abogada graduada en la Universidad Técnica Particular de Loja. Coordinadora de prácticas pre profesionales, Universidad Católica de Cuenca. Cursando Maestría en Diagnóstico Clínico y Biología Molecular, Universidad Católica de Cuenca, Ecuador.

JG: Docente de la Universidad Católica de Cuenca, facultad de medicina. 4to nivel en Microbiología, expositor de congresos. Universidad Católica de Cuenca, Ecuador.

SP: Doctor en Medicina y CirugíaUniversidad se Cuenca. Especialista en Medicina Interna- Universidad del Azuay. Especialista en Docencia UniversitariaUniversidad Católica de Cuenca. Master en Endocrinología Avanzada-Universidad de Alcalá Madrid. Especialista en Medicina Alcalá Madrid. Especialista en Medicina Interna- Universidad de Cuenca. Doctor en Universidad Católica de Cuenca, Ecuador. 
VM: Química Farmaceuta, Universidad Católica de Cuenca Abogada graduada en la Universidad Técnica Particular de Loja. Coordinadora de prácticas pre profesionales, Universidad Católica de Cuenca. Cursando Maestría en Diagnóstico Clínico y Biología Molecular, Universidad Católica de Cuenca, Ecuador.

JG: Docente de la Universidad Católica de Cuenca, facultad de medicina. 4to nivel en Microbiología, expositor de congresos. Universidad Católica de Cuenca, Ecuador.

SP: Doctor en Medicina y CirugíaUniversidad se Cuenca. Especialista en Medicina Interna- Universidad del Azuay. Especialista en Docencia UniversitariaUniversidad Católica de Cuenca. Master en Endocrinología Avanzada-Universidad de Alcalá Madrid. Especialista en Medicina Interna- Universidad de Cuenca. Doctor en Ciencias Médicas- Universidad del Zulia. Universidad Católica de Cuenca, Ecuador.

\begin{abstract}
RESUMO
Escherichia coli (E. coli) faz parte do grupo das principais enterobactérias, que por sua alta prevalência e capacidade de gerar resistência a certos antimicrobianos é causa de diversos processos infecciosos de origem comunitária, principalmente infeccões do trato urinário. 0 mecanismo de defesa dessas bactérias é a ativação enzimática por meio de Betalactamas do tipo ESBL, AmpC e CARBAPENEMASES. Materiais e Métodos. Desenho documental transversal, com abordagem quantitativa, a população foi constituída por 671 laudos de urocultura processados no período de janeiro a abril de 2020 no Laboratório Clínico Neolab da cidade de Cuenca no Equador. Resultados. $96,4 \%$ das amostras são do sexo feminino e $3,6 \%$ do sexo masculino, idade média, o estudo teve maior prevalência em pacientes com 40 anos de idade. 7,62\% das amostras foram ESBL positivas, 0,13\% AmpC e 0\% CARBAPENEMASES. Os antibióticos com maior taxa de eficácia para o tratamento de E. coli produtoras de ESBL em urocultura foram nitrofurantoína, gentamicina e fosfomicina, com taxas de eficácia de $87,93 \%, 79,31 \%$ e $70,68 \%$, respectivamente. No entanto, antimicrobianos como a Ampicilina apresentaram resistência de $100 \%$ e Trimetoprim sulfametoxazol de 72,41\%. Conclusão. A detecção da frequência das enzimas ESBL, AmpC e Carbapenemases orienta um tratamento terapêutico adequado. Pacientes com bactérias produtoras de ESBL apresentam alta taxa de mortalidade quando tratados com antimicrobianos que as bactérias apresentam alta resistência.
\end{abstract}

Palavras-Chave: Infecções urinárias; Resistência bacteriana a medicamentos; Teste de sensibilidade microbiana; Infecções adquiridas na comunidade; enzimas

\section{INTRODUCCIÓN}

$\mathrm{L}$ a infección por Escherichia coli (E.coli) causa una alerta mundial en la actualidad, cada vez aumenta el número de pacientes infectados con esta bacteria. A esto se suma la resistencia a los antimicrobianos y los mecanismos de defensa que desarrollan las bacterias frente a los antibióticos, con la consecuente aparición de microorganismos multirresistentes (1).

Cada vez aparecen nuevas cepas de $E$. coli, productoras de betalactamasas de espectro extendido (BLEE); Betalactamasas de tipo AmpC y carbapenemasas, las cuales inhiben la acción a varios fármacos, especialmente los Betalactámicos (2). Hasta finales de la década de 1990, las enterobacterias principalmente Klebsiella pneumoniae, productoras de BLEE de tipo SHV-1 y TEM-1, TEM-2 las cuales fueron responsables de infecciones nosocomiales graves. Sin embargo, esta escena ha cambiado significativamente, la $E$. coli productora de betalactamasas de espectro extendido de tipo CTX-M, se ha diseminado en todo el mundo siendo una causa importante de infecciones de inicio comunitario, principalmente infecciones del tracto urinario (ITU) (3).

La aparición y propagación mundial de la enzima CTX-M-15 ha cambiado la visión sobre la epidemiología de la $E$. coli productora de BLEE. Es así que, actualmente este tipo de betalactamasa se encuentra en la mayoría de las regiones a nivel mundial (4).

En América del Norte se reporta betalactamasa de tipo CTX-M-15, con una incidencia mínima. Sin embargo, Estados Unidos refleja una elevada prevalencia en $E$. Coli productora de esta enzima $(5,6)$.

Según el Sistema Europeo de Vigilancia de Resistencia a los Antibióticos (EARSS), la resistencia a las cefalosporinas de tercera generación, se debe principalmente a la producción de BLEE de tipo CTX-M-15 (7).

Las AmpC se han descrito en algunas especies, entre estas destaca la E. coli con una relevancia tanto epidemiológica como 
clínica, dando lugar a fracasos terapéuticos, además las AmpC de tipo plasmáticas tienen gran capacidad para movilizarse, constituyendo un importante reservorio para un amplio rango de infecciones nosocomiales (8).

Las bacterias productoras de carbapenemasas se reportan por primera vez en Latinoamérica, en la década de los 80 (9).

En un estudio realizado en Ecuador en la ciudad de Cuenca, en el Hospital José Carrasco Arteaga en el año 2016, se encontró que estas bacterias eran muy prevalentes en pacientes que se encontraban en UCI (Unidades de Cuidados Intensivos), y su índice de mortalidad era del 40-50\% (10).

Cada vez es más frecuente el incremento de las infecciones por cepas siendo $E$ coli, la principal causa de de infección de tracto urinario (ITU) (11). Lo que se convierte en un grave problema para nuestra sociedad, ya que complica el tratamiento antimicrobiano en pacientes portadores de estas cepas. Razón por la cual en el presente estudio se detectaron las enzimas (BLEE) (AmpC) y carbapenemasas, de E. coli aisladas de muestra de urocultivo durante el período de enero-abril 2020, de origen comunitario, se debe realizar un seguimiento de datos mínimo una vez cada 6 meses.

\section{MATERIALES Y MÉTODOS}

$\mathrm{L}$ a investigación es de tipo positivista con un enfoque cuantitativo, de diseño documental, de corte transversal descriptiva. La población objeto de estudio, estuvo comprendida por 761 aislados clínicos procedentes de urocultivo identificados con E.coli. Se realizó un muestreo no probabilístico-intencional. Los datos fueron recopilados de fuentes secundarias ingresados en la base de datos del departamento de microbiología del Laboratorio clínico NEOLAB de la ciudad de Cuenca, comprendidos en el periodo eneroabril de 2020.

Criterios de Inclusión. Registros de urocultivo de origen comunitario con aislamiento de E. coli y que presentaran resistencia al menos a un antimicrobiano. Registros de urocultivos que contengan la información completa las cuales contengan las variables objeto de estudio.

Criterios de Exclusión. Registros de urocultivo donde se aíslan otros agentes causales, muestras contaminadas, pacientes con formularios incompletos.

Los datos recolectados fueron ingresados en una matriz de análisis que contiene las siguientes variables: Sexo, edad, susceptibilidad antimicrobiana y mecanismo de resistencia.

\section{Procedimiento}

El método de medición usado en el laboratorio clínico fue el método KirbyBauer de difusión en agar, según los lineamientos establecidos por el CLSI 2020 (12).

El proyecto se fundamentó con principios éticos, basados en la Declaración de Helsinki Adendum de Taiwán 2016, dando una protección y privacidad a los datos de los pacientes que han acudido exámenes clínicos, objeto de estudio, serán manejados con estricta confidencialidad, no se vulnerará el derecho de ningún paciente, el nombre del paciente no será mencionado, únicamente se tomara en cuenta la edad, el sexo, y los datos como nombres o su identidad dejando en anonimato a las personas de este estudio, además dicha 
información no se utilizará con otros fines que no sean de carácter investigativo.

Para el análisis estadístico se generó una base de datos en el programa Excel 2013 y para el análisis estadístico se empleó el SPSS versión 20.0. Se llevó a cabo mediante estadística descriptiva, medidas de tendencia central y medidas de tendencia no central, análisis de frecuencia. Para la presentación de resultados se usaron tablas y los gráficos se representaron mediante el empleo de gráficos de barras.

\section{RESULTADOS Y DISCUSIÓN}

$\mathrm{E}$ n la Tabla 1 del total de muestras revisadas en el presente estudio el 96.4\% pertenecieron al género femenino y el $3.6 \%$ al género masculino. El valor de $p$, es muy significativo, demostrando una relación entre el grupo etario y E. coli.

El promedio de edades de nuestra población es de 40 años es decir 21.5 años. La población de negativos para $E$. coli es de 49.2 años, mientras que para positivos en $E$. coli es de 39 años, también existe una relación entre Grupo etarios dicotomizados según adultos y demás grupos.

El OR es de 0.70, demostrando que una persona adulta, que no pertenece al grupo de adulto mayor ni adolescencia - juventud tiene 0.7 más posibilidades de desarrollar infección por E. coli.

Una persona de sexo femenino tiene 3.54 más posibilidades de desarrollar infección por E. coli que una persona de sexo masculino.

Tabla 1. Frecuencia y porcentaje de E.coli en ITU, según el grupo etario y sexo

\begin{tabular}{|c|c|c|c|c|c|c|}
\hline & & & E.C & & & \\
\hline VARIABL & & NEG & & POS & & \\
\hline & & $\mathbf{n}$ & $\%$ & n & $\%$ & p. \\
\hline GRUPOS & 0-5 AÑOS & 15 & 3,5 & 21 & 6,3 & 0.002 \\
\hline ETARIOS & 6 - 11 AÑOS & 14 & 3,3 & 20 & 6,0 & \\
\hline & 12-18 AÑOS & 22 & 5,1 & 12 & 3,6 & \\
\hline & 19-26 AÑOS & 59 & 13,8 & 33 & 9,9 & \\
\hline & 27-59 AÑOS & 251 & 58,6 & 166 & 49,8 & \\
\hline & $\begin{array}{l}60 \text { AÑOS O } \\
\text { MAS }\end{array}$ & 67 & 15,7 & 81 & 24,3 & \\
\hline SEXO & MASCULINO & 50 & 11,7 & 12 & 3,6 & 0,000 \\
\hline & FEMENINO & 378 & 88,3 & 321 & 96,4 & \\
\hline
\end{tabular}

La media de la edad de la población total: 40,1 $\pm 21,5$; Media edad población negativos para E. coli: 49,2 $\pm 28,8$; Media edad población positivos para E. coli: $39,3 \pm$
20,6; Grupo etarios dicotomizados según adultos y demás grupos etarios: $\mathrm{OR}=0,70$, IC $(0,53-0,94)$, p. 0,016 ; Sexo: $\mathrm{OR}=3,54$, IC $(1,85-6,76)$, p. 0,000 . 
En la Tabla 2, se puede observar que del total de muestras positivas a E. coli, 58 fueron $B L E E$ siendo un $7.6 \%$, una muestra portadora de $A m p C$ que representa el $0.1 \%$ y no se obtuvieron muestras positivas a carbapenemasas.

Tabla 2. Enzimas de resistencia presentes en E.coli

\begin{tabular}{llrr}
\hline & BLEE & $\boldsymbol{n}$ & \multicolumn{1}{c}{$\%$} \\
\hline \multirow{2}{*}{ TODOS } & AmPC & 58 & 7,6 \\
& CARBAPENENSAS & 1 & 0,1 \\
& NINGUNA & 0 & 0,0 \\
DX POSITIVO E. COLI & ATEE & 702 & 92,2 \\
& CARBAPENENSA & 58 & 17,4 \\
PRESENCIA DE ENZIMAS & BLEE & 1 & 0,3 \\
DE RESISTENCIA & AMPC & 0 & 0,0 \\
\hline
\end{tabular}

En el Gráfico 1, los antibióticos con un mayor índice de efectividad para tratar E.coli productora de BLEE en urocultivos son la Nitrofurantoína, la Gentamicina y Fosfomicina con índices de efectividad del
$87.93 \%, \quad 79,31 \% \quad y \quad 70,68 \%$ respectivamente. Sin embargo los antimicrobianos como Ampicilina presentan una resistencia del $100 \%$ y Trimethoprim sulfa metoxazol del $72.41 \%$.

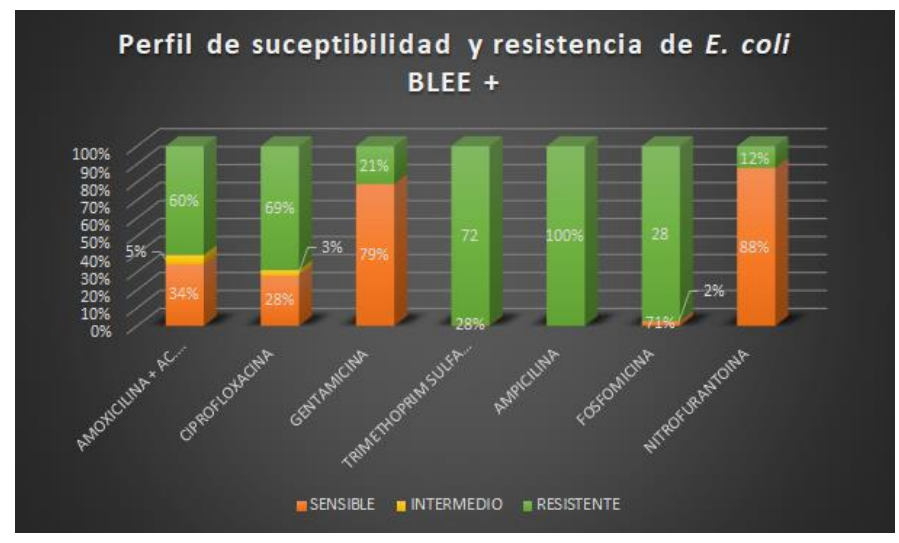

Gráfico 1. Perfil de sensibilidad y resistencia de los diferentes antimicrobianos utilizados para tratar E. coli productora de BLEE en pacientes de consulta externa en el Laboratorio Clínico Neolab en el período de enero-abril 2020. (Cualquier enterobacteria con agrandamiento del halo de inhibición de cefalosporina de tercera generación hacia el disco de Amoxicilina + ac. clavulánico, independientemente de la zona de inhibición de las cefalosporina de tercera generación implica producción de BLEE, informando resistencia a todas las penicilinas, cefalosporina y monobactams). 
Seguidamente, Gráfico 2, se muestra que los antibióticos ciprofloxacina y nitrofurantoína son los únicos sensibles para tratar E. coli que produce la enzima AmpC, dentro de nuestro estudio. Esto coinciden con los estudios revisados los más efectivos para tratar infecciones del tracto urinario causadas por E.coli que llegasen a producir las enzimas BLEE, AmpC y CARBAPENEMASAS.

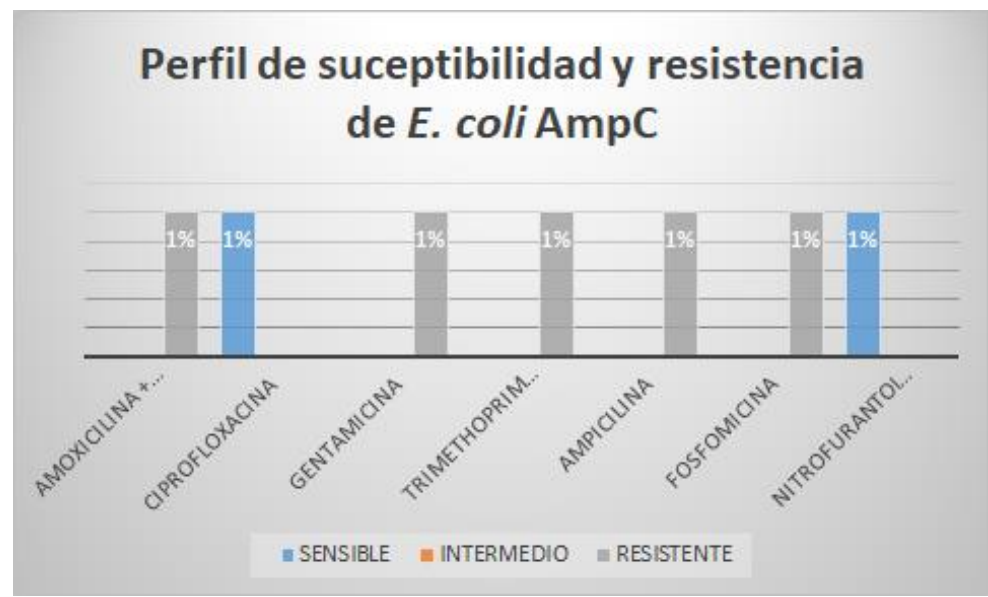

Gráfico 2. Perfil de sensibilidad y resistencia de los diferentes antimicrobianos utilizados para tratar E. coli productora de AmpC en pacientes de consulta externa en el Laboratorio Clínico Neolab en el período de enero-abril 2020. (Beta lactamasas de tipo AmpC: La resistencia a cefoxitina puede ser un indicador, test de doble disco muestra achatamiento del halo de inhibición entre un buen y mal inductor).

\section{DISCUSIÓN}

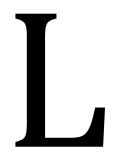

a E. coli como causante de las infecciones del tracto urinario (ITU) ha sido ampliamente descrita a nivel mundial, al igual que aquellas que producen las beta-lactamasas de espectro extendido (BLEE), las AmpC y CARBAPENEMASAS, con la diferencia que las AmpC son otro tipo de betalactamasas que no poseen en la actualidad un método estandarizado por el Instituto de Estándares de Laboratorios Clínicos (CLSI) para su detección fenotípica (15). Durante los últimos años, se ha reportado un número creciente de estudios que demuestran una alta prevalencia de estos microorganismos aislados no únicamente de origen intrahospitalario, sino también de origen comunitario (16).
Resulta compleja la comparación de prevalencias entre estudios debido a que todos tienen diferentes criterios de inclusión, características y tipos de muestras. Así como el lugar de proveniencia de las mismas (17). Sin embargo, en el presente estudio de 761 muestras analizadas durante el período de enero - abril de 2020 que fueron de origen comunitario realizados en el Laboratorio Clínico Neolab de la ciudad de Cuenca, es muy superior a las frecuencias reportadas en Europa que indican tan solo un $0,14 \%$ de 7054 muestras haciendo referencia a productoras de BLEE. Siendo un porcentaje muy bajo que pudo variar los métodos de detección (4). 
En Rajasthan, en la India se encontró una prevalencia de BLEE de $66,9 \%$, y de betalactamasas AmpC de 3,5\% (18) y en EE.UU. mostraron un resultado de $1,2 \%$ de E. coli productores de AmpC (19). Al igual que estudios de frecuencia de enzimas AmpC en pacientes de origen comunitario realizados en EE.UU. Se encontró una frecuencia de $0,6 \%$ y $0,5 \%$ en E. coli (20). En otro estudio realizado en España se detectó que la prevalencia de AmpC fue del 0,17 (21), y en Argentina se encontró un porcentaje del 0,55\% de E.coli productoras de AmpC (22). Por otra parte, en Colombia la frecuencia es de 2,05\% de AmpC (23). Las frecuencias de prevalencia de AmpC en la revisión realizada de varios artículos coinciden con nuestro estudio ya que no sobrepasan el 3\% lo que es muy bueno ya que seguirá habiendo opciones de tratamiento para pacientes con ITU causado por E.coli.

Es indiscutible la relevancia clínica que tienen las betalactamasas AmpC más aún cuando se encuentran mecanismos de resistencia asociados como por ejemplo en un estudio de Caracas en el 2009 donde AmpC amplían el rango de acción hidrolítica hasta los carbapenémicos, o aquellas AmpC de espectro extendido que tienen una resistencia a cefalosporinas de cuarta generación (24).

En este estudio el aislamiento de Escherichia coli productora de BLEE en su mayoría afectó al género femenino $(91,85 \%)$ y a todos los grupos etarios dentro de este género, especialmente los comprendidos entre 27-59 años. Esto se debe a que existen cambios en la flora vaginal debido a la disminución de estrógenos post - menopausia. En cuanto que en los hombres el crecimiento prostático es un factor habitual sobre todo en el hombre anciano causando retención de orina desencadenando infecciones del tracto urinario. Elevando así la frecuencia con la que pacientes en estas edades están propensos a desarrollar ITU causado por $E$. coli (14).

En la ciudad de Cuenca se realizó un estudio similar, el cual reporta que 103 aislamientos de E.Coli el $6.8 \%$ fueron productoras de BLEE lo que se asemeja muchos a resultados, manteniéndose en estos valores por varios años (25).

El mejor tratamiento de una ITU por gérmenes productores de BLEE es la prevención y en ella se incluye evitar el uso de cefalosporinas de tercera generación y de fluoroquinolonas en el tratamiento empírico de las ITU a nivel comunitario. En España el tratamiento de elección de la ITU por BLEE en la comunidad son la fosfomicina-trometamol y la nitrofurantoína (tasas de sensibilidad de E.coli del $97 \%$ y el $94 \%$ respectivamente). Lo que coincide sobre todo en el uso de nitrofurantoína y la fosfomicina con nuestro estudio en el cual los índices de sensibilidad fueron del $87,93 \%$ y el $79,31 \%$. Siendo la principal diferencia con este estudio la Sensibilidad a la Gentamicina (26).

Los betalactámicos y las quinolonas son los antibióticos de primera línea en el tratamiento de las ITU por E.coli en todos los países de Sudamérica, incluyendo aquellos que siguen las recomendaciones de la Sociedad Americana de Enfermedades Infeccionas (IDSA) EN nuestro estudio encontramos resistencia del $100 \%$ en el caso de la ampicilina y de Trimethoprim sulfa metoxazol del $72.41 \%$ (27).

En otro estudio realizado en México se recomienda como opción terapéutica la nitrofurantoína 50 mg o $100 \mathrm{mg}$ una vez al día, fosfomicina trometamol $3 \mathrm{~g}$ cada diez 
días, trimetoprim 100 mg una vez al día y durante el embarazo cefalexina $125 \mathrm{mg}$ o $250 \mathrm{mg}$ o cefaclor $250 \mathrm{mg}$ una vez al día (11).

\section{CONCLUSIONES}

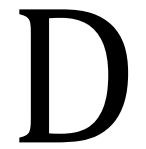
etectar la frecuencia de enzimas BLEE, AmpC y Carbapenemasas, es importante para orientar adecuadamente a un tratamiento terapéutico. Los pacientes con bacterias productoras de BLEE, tienen un alto índice de mortalidad al ser tratados con antimicrobianos que las bacterias tengan resistencia alta. Lo que se hace mención a la importancia médica al momento de tratar infecciones en especial las que tengan microorganismos multirresistentes.

La edad donde mayor contagio de infecciones por E. Coli es en mujeres de 27 a 49 años de sexo femenino. Los antibióticos con un mayor índice de efectividad para tratar E.coli productora de BLEE en urocultivos son la Nitrofurantoína, Gentamicina y Fosfomicina 87.93\%, 79,31\% y 70,68\% respectivamente. Sin embargo los antimicrobianos como Ampicilina presentan una resistencia del $100 \%$ y Trimethoprim sulfa metoxazol $72.41 \%$. Se muestra que los antibióticos ciprofloxacina y nitrofurantoína son los únicos sensibles para tratar E. coli que produce la enzima $\mathrm{AmpC}$,

Cada vez, cobra mayor importancia el educar a la población y las empresas farmacéuticas a un uso racional de antimicrobianos para evitar que estas creen otros mecanismos de resistencia y dificulte el tratamiento antibacteriano.

De igual manera no se puede descartar la importancia de realizar este tipo de estudios, controlando la resistencia antimicrobiana ya que desde un punto de vista epidemiológico. Lo cual, ayudaría a dar pautas de tratamiento para diferentes enfermedades como las del tracto urinario.

\section{REFERENCIAS BIBLIOGRÁFICAS}

1. Peirano G, Pitout JDD. Molecular epidemiology of Escherichia coli producing CTX-M $\beta$-lactamases: the worldwide emergence of clone ST131 025:H4. Int J Antimicrob Agents. 2010;35(4):316-21 DOI.10.1016/j.ijantimicag.2009.11.003.

2. Guevara LM, Chilón S. Resistencia enzimática a betalactámicos en Escherichia coli aisladas de pacientes atendidos en el hospital regional docente de Cajamarca. UPAGU. (2020, VII); Perú. Disponible en: http://repositorio.upagu.edu.pe/bitstream /handle/UPAGU/1290/FYB-0112020.pdf? sequence $=1$ \&isAllowed $=y$

3. Oliver A, Cantón R. Enterobacterias Productoras De B-Lactamasas Plasmídicas De Espectro Extendido. Control Calid SEIMC. 1990;1-10. Disponible en: https://www.seimc.org/contenidos/ccs/r evisionestematicas/bacteriologia/Blees.pd $\mathrm{f}$

4. Morejón M. Betalactamasas de espectro extendido. Rev Cubana Med. 2013;52(4):272-80. Disponible en: http://scielo.sld.cu/scielo.php?script=sci_a rttext\&pid=S0034-75232013000400006

5. McGettigan SE, Hu B, Andreacchio K, Nachamkin I, Edelstein PH. Prevalence of CTX-M $\beta$-lactamases in Philadelphia, Pennsylvania. J Clin Microbiol. 2009;47(9):2970-4. DOI: 10.1128 / JCM.00319-09

6. Sidjabat HE, Paterson DL, AdamsHaduch JM, Ewan L, Pasculle AW, Muto CA, et al. Molecular epidemiology of CTX-Mproducing Escherichia coli isolates at a tertiary medical center in Western Pennsylvania. Antimicrob Agents Chemother. 2009;53(11):4733-9. DOI:10.1128/AAC.00533-09

7. Lau SH, Kaufmann ME, Livermore DM, Woodford N, Willshaw GA, Cheasty T, et al. UK epidemic Escherichia coli strains A-E, 
with CTX-M-15 $\beta$-lactamase, all belong to the international 025:H4-ST131 clone. J Antimicrob Chemother. 2008;62(6):12414. Disponible en: http://portal.amelica.org/ameli/jatsRepo/ 50/50602013/html/index.html

8. Calvo J, Cantón R, Fernández F, Mirelis B, Navarro F. Detección fenotípica de mecanismos de resistencia en gramnegativos, SEIMC. Seimc. 2011. 54 p. Disponible en: https://www.seimc.org/contenidos/docu mentoscientificos/procedimientosmicrobi ologia/seimc-

procedimientomicrobiologia38.pdf

9. Queenan AM, Bush K. Carbapenemases: The versatile $\beta$-lactamases. Clin Microbiol Rev. 2007;20(3):440-58.DOI 10.1128 / CMR.00001-07

10. Ríos VE. Prevalencia y factores asociados a mortalidad por sepsis en pacientes de cuidados intensivos en el Hospital José Carrasco Arteaga. Cuenca, 2012-2015 [master's thesis on the Internet]. Cuenca; 2018-11-11 [cited 26 Mar. 2021]. Disponible en: http://dspace.ucuenca.edu.ec/handle/123 $456789 / 30478$.

11. Guzmán, N, and Herney A: García-P. "Novedades en el diagnóstico y tratamiento de la infección de tracto urinario en adultos." Revista Mexicana de Urología 80.1 (2020): 1-14.Disponible en: https://www.medigraphic.com/pdfs/uro/ ur-2020/ur201f.pdf

12. Kahlmeter G, Giske CG, Kirn TJ, Sharp SE. Point-counterpoint: Differences between the European Committee on Antimicrobial susceptibility testing and Clinical and Laboratory standards institute recommendations for reporting antimicrobial susceptibility results. J Clin Microbiol. 2019;57(9):1-6. DOI: 10.1128 / JCM.01129-19

13. Ermakova EI. Recurrent urinary tract infections in women. Akusherstvo $\mathrm{i}$ Ginekol (Russian Fed. 2020;2020(7):1518. DOI: 10.1097 / 00001703-20021000000016
14. Echevarria Zárate JI, Osores Plenge FL. Infección del tracto urinario y manejo antibiótico. Acta Médica Peru. 2006;23(1):26-31. Disponible en: http://www.scielo.org.pe/scielo.php?scrip $\mathrm{t}=$ sci_arttext\&pid=S1728-

59172006000100006

15. Sociedad Venezolana de Microbiología. DDVM. Betalactamasas tipo AmpC: generalidades y métodos para detección fenotípica. Rev la Soc Venez Microbiol [Internet]. 2005;29(2):78-83. Available from: http://www.redalyc.org/exportarcita.oa?i $\mathrm{d}=199414957003$

16. Perozo Mena AJ, Castellano González MJ. Detección de betalactamasas de espectro extendido en cepas de la familia enterobacteriaceae. Kasmera. 2009;37(1):25-37. Available from: http://ve.scielo.org/scielo.php?pid=S0075

52222009000100004\&script=sci_abstract 17. Bonnet R, Sampaio JLM, Labia R, De Champs C, Sirot D, Chanal C, et al. A novel CTX-M $\quad \beta$-lactamase (CTX-M-8) in cefotaxime-resistant Enterobacteriaceae isolated in Brazil. Antimicrob Agents Chemother. 2000;44(7):1936-42. DOI: 10.1128 / aac.44.7.1936-1942.2000

18. Dalela G, Sweta G, Jain DK, Mehta P. Antibiotic resistance pattern in uropathogens at a tertiary care hospital at jhalawar with special reference to ESBL, AmpC $\beta$-lactamase and MRSA production. J Clin Diagnostic Res. 2012;6(4 SUPPL. 2):645-51. Disponible en: https://www.jcdr.net/articles/pdf/2117/ 25\%20-\%204201.pdf

19. Deshpande LM, Jones RN, Fritsche TR, Sader HS. Occurrence of plasmidic AmpC type $\beta$-lactamase-mediated resistance in Escherichia coli: report from the SENTRY Antimicrobial Surveillance Program (North America, 2004). Int J Antimicrob Agents. 2006;28(6):578-81. DOI: 10.1128 / CMR.00036-08

20. Hanson ND, Moland ES, Hong SG, Propst K, Novak DJ, Cavalieri SJ. 
Surveillance of community-based reservoirs reveals the presence of CTX-M, imported $\mathrm{AmpC}$, and OXA-30 $\beta$-lactamases in urine isolates of Klebsiella pneumoniae and Escherichia coli in a U.S. community. Antimicrob Agents Chemother. 2008;52(10):3814-6. DOI: 10.1128 / AAC.00877-08

21. Navarro-Navarro $M$, Robles-Zepeda RE, Garibay-Escobar A, Ruiz-Bustos E. Escherichia coli y Klebsiella pneumoniae comunitarias y hospitalarias productoras de $\beta$-lactamasas en hospitales de Hermosillo, Sonora. Salud Pública Mex. 2011;53(4):341-4. Disponible en: https://www.medigraphic.com/pdfs/salp ubmex/sal-2011/sal114h.pdf

22. Jure MA, Presti C, Cudmani NM, Grellet LM, López C, Musa EH, et al. $\beta$ lactamasas AmpC plasmídicas tipo CMY-2 emergentes en Tucumán, Argentina. Rev Argent Microbiol. 2011;43(1):24-7. Disponible en: https://seq.es/seq/02143429/25/2/seral.pdf

23. Morales GI, Bolaños Contreras CC, Larrazábal Ruiz TJ. Enterobacterías aisladas en un centro hospitalario de la ciudad de Valledupar y frecuencia de betalactamasas de espectro extendido y betalactamasas inducibles. Biociencias [Internet]. 1 de diciembre de 2011 [citado 27 de marzo de 2021];6(2):33-40. Disponible en: https://revistas.unilibre.edu.co/index.php /biociencias/article/view/2778

24. Martínez DV. Betalactamasas tipo AmpC: Generalidades y métodos para detección fenotípica. Rev. Soc. Ven. Microbiol. [Internet]. 2009 Dic [citado 2021 Abr 25];29(2):78-83. Disponible en: http://ve.scielo.org/scielo.php?script=sci_ arttext\&pid=S131525562009000200003\&lng=es

25. León Cajamarca PA, Vázquez Guillén GB. Prevalencia de cepas de escherichia coli productoras de betalactamasas de espectro extendido (blee) en muestras de orina de pacientes ambulatorios de los Centros de Salud 1, 2 y 3 de la ciudad de Cuenca [bachelor's thesis on the Internet]. Cuenca; 2013 [cited 26 Mar. 2021]. Available from: http://dspace.ucuenca.edu.ec/handle/123 456789/4631

26. Palou J, Pigrau C, Molina I, Ledesma JM, Angulo J. Etiología y sensibilidad de los uropatógenos identificados en infecciones urinarias bajas no complicadas de la mujer (Estudio ARESC): implicaciones en la terapia empírica. Med Clin (Barc). 2011;136:1-7. [DOI 10.1016/j.medcli.2010.02.042] [Consulta: 23/04/2014]

27. Gonzales D, Jaulis J, Tapia E, Samalvides C. Sensibilidad antibiótica de bacterias causantes de infecciones del tracto urinario en un hospital general: Enero - junio del año 2008. Rev Med Hered [Internet]. 2009 En el [citado $2021 \mathrm{Abr}$ 25];20(1):11-15. Disponible en:http://www.scielo.org.pe/scielo.php?sc ript=sci_arttext\&pid=S1018130X2009000100004\&lng=e

Conflicto de intereses: Los autores declaran que no existe conflicto de intereses para la publicación del presente artículo científico.

Financiamiento: Los autores autofinanciaron Agradecimientos: Los autores no declaran 